\title{
Prognostic implications of phosphatidylinositol 3-kinase/AKT signaling pathway activation in gastric carcinomas
}

\author{
Paula Blandina Ola Chiappini ${ }^{1}$, Ivan Ucella Dantas de Medeiros ${ }^{1}$, Luiz Guilherme Cenaglia Lima ${ }^{1}$, \\ Jose Humberto Fregnani ${ }^{2}$, Suely Nonogaki ${ }^{1}$, Wilson Luiz da Costa $\mathrm{Jr}^{1}$, Felipe Jose Fernandez Coimbra ${ }^{1}$, \\ Milton Jose de Barros e Silva ${ }^{1}$, Celso Abdon Lopes de Melloํㅜ, Clovis Antonio Lopes Pinto', \\ Maria Dirlei Begnami ${ }^{1}$
}

${ }^{1} \mathrm{AC}$ Camargo Cancer Center, São Paulo, Brazil
${ }^{2}$ Hospital de Cancer de Barretos, Barretos, Brazil

Submitted: 8 September 2015

Accepted: 20 January 2016

Arch Med Sci 2017; 13, 6: 1262-1268

DOI: 10.5114 /aoms.2016.60394

Copyright () 2016 Termedia \& Banach
Corresponding author: Maria Dirlei Begnami $\mathrm{MD}, \mathrm{PhD}$

AC Camargo Cancer Center Rua Antonio Prudente, 211 01509010 Sao Paulo, Brazil Phone: +551121895103 E-mail: mariadirlei@gmail. com

\begin{abstract}
Introduction: Activation of the phosphatidylinositol 3-kinase (PI3K)/AKT pathway plays a critical role in carcinogenesis and resistance to anticancer drugs. In this study, gastric carcinomas (GC) were investigated and statistical analyses were performed concerning the correlation between the clinicopathological features and activation of the PI3K/AKT pathway.

Material and methods: Immunohistochemistry for p-AKT, p-mTOR and PTEN was performed in 239 GC and 200 non-neoplastic gastric tissues. The clinicopathological parameters were recorded from the medical charts. Statistical significance was defined by a $p$-value $<0.05$.

Results: High p-AKT expression was observed in $10 \%$ of the normal gastric tissue and in $90 \%$ of GC, and it was significantly associated with tumor size $(p<0.001)$, T3/T4 tumors $(p<0.001)$, and presence of metastases $(p=0.02)$. Similarly, $p$-mTOR positivity was found in GC cells, but not in the normal gastric mucosa, and was correlated with perineural invasion $(p=0.02)$ and T3/T4 tumors $(p=0.03)$. On the other hand, PTEN expression was weak and focal in the tumor cells, while in the normal gastric tissue this staining was strong and diffuse. Importantly, the expression of p-mTOR and PTEN was associated with overall survival.

Conclusions: The results of the present study, characterized by the loss of PTEN expression and higher expression of $p$-AKT and p-mTOR in the majority of tumor cells, apparently are implicated in the carcinogenesis and progression of GC. The identification of p-mTOR and PTEN expression as prognostic factors corroborates the identification and use of potential target drugs that could be more efficient for the treatment of these patients.
\end{abstract}

Key words: gastric carcinomas, PI3K/AKT pathway, molecular markers.

\section{Introduction}

Gastric carcinoma (GC) is one the most aggressive tumors of the gastrointestinal tract. The prognosis remains poor, even for patients surgically treated with complete tumor resection [1]. Despite improvements in cancer therapy over the past 20 years, survival of patients with advanced and metastatic GC is still measured in months. Gastric carcino- 
genesis has been associated with multiple genetic and epigenetic alterations. Also various gene abnormalities have been associated with the maintenance of the malignant phenotype and tumor cell survival [2]. Recently, the PI3K/AKT signaling pathway has risen to prominence as a key regulator for cell cycle proliferation, growth, survival, protein synthesis, and glucose metabolism [3]. This molecular pathway has been frequently inappropriately activated in many solid tumors, and there is evidence showing that some components of this pathway can be used as molecular cancer targets $[4,5]$.

In this study, we performed correlations between the protein expressions of PTEN, p-AKT, and p-mTOR and clinicopathological parameters in 239 GC patients.

\section{Material and methods}

\section{Patients}

From 2000 to 2010, 239 patients with GC (144 males and 95 females, mean age: 65 years, range: 20-88 years) were treated by surgery (total or subtotal gastrectomy with lymphadenectomy) at the Department of Abdominal Surgery, AC Camargo Cancer Center, Sao Paulo, Brazil. The surgical specimens were fixed in $10 \%$ formaldehyde and routinely processed for paraffin embedding. Histological sections $(4 \mu \mathrm{m})$ were stained with hematoxylin and eosin and representative tumor sections were subjected to immunohistochemistry. None of the patients received preoperative therapy. The clinicopathological parameters were recorded from the medical charts. Follow-up data were available for all patients. Two hundred biopsies of gastric tissues obtained from patients without GC were used as normal controls. The Human Ethics Review Committee of the AC Camargo Cancer Center approved this study (number 122/09). A signed informed consent form was obtained from each patient. All of the specimens were handled and made anonymous according to ethical and legal standards.

\section{Immunohistochemistry procedures and staining evaluation}

The $4 \mu \mathrm{m}$ sections of the GC and normal gastric tissue were placed on adhesive-coated slides for immunohistochemistry. The sections were deparaffinized with xylene and dehydrated through a series of graded alcohols. Microwave antigen retrieval was used and consisted of placing the slides in $10 \mathrm{mM}$ citrate buffer ( $\mathrm{pH}$ 6.0) for $15 \mathrm{~min}$. A standard peroxidase-conjugated streptavidinbiotin method was used to detect the staining reaction (Advance, HRP Link, DAKO, Carpinteria, USA). The sections were stained with the follow- ing primary antibodies: phospho mTOR (p-mTOR, Ser2448; Cell Signaling Technology, Danvers, MA, USA; dilution 1 : 50), phospho AKT (p-AKT, Ser473, Cell Signaling Technology, Danvers, MA; dilution 1 : 200), and PTEN (rabbit polyclonal; Abcam, Fremont, CA; dilution 1 : 100). External positive control tissues included breast carcinomas positive for the antibodies studied. For negative controls, non-immunized mouse or rabbit IgG serum (Vector Laboratories, Burlingame, CA) was used as the primary antibody.

Two pathologists using optical microscopy (M.D.B. and C.A.L.P.) analyzed the slides. Tumors containing $>50 \%$ positive tumor cells were classified as high expression and those with $\leq 50 \%$ positive tumor cells as low expression.

\section{Statistical analysis}

The statistical analyses were performed using the SPSS (Statistical Package for the Social Sciences) software version 15.0 (SPSS Inc., Chicago, IL, USA) and MedCalc version 11. Correlations between immunohistochemical expression and clinicopathological findings were estimated by Pearson correlation. Either the $\chi^{2}$ or Fisher's exact test (two-sided) was used to determine correlations between protein expression and clinicopathological parameters. Comparison between groups was performed using the Mann-Whitney $U$ or KruskalWallis test. Actual survival rates were estimated using the Kaplan-Meier method, and differences in survival curves were analyzed using the logrank test. We used the Cox proportional hazards model to identify independent risk factors for death. Significance was defined by a $p$-value less than 0.05 .

\section{Results}

\section{Analysis of clinicopathological features}

The clinicopathological features of the GC patients are summarized in Table I. The tumor size ranged from 0.45 to $19 \mathrm{~cm}$ (mean: $5 \mathrm{~cm}$ ). The median follow-up period of the patients was 49 months (range: 1-154 months). Recurrent disease was diagnosed in 81 (34\%) patients, and disease-related death occurred in 154 (64.4\%) patients.

\section{Immunohistochemical expression of p-AKT, p-mTOR, and PTEN in GCs and normal gastric mucosa}

p-AKT showed positive staining in the nuclei and cytoplasm either in the tumor cells or in the normal gastric tissue, whereas the surrounding stromal cells showed little or no staining. High p-AKT expression was observed in $90 \%$ of the tumor samples and in only $10 \%$ of the normal gas- 
Table I. Clinicopathological characteristics of the 239 gastric carcinomas

\begin{tabular}{|c|c|c|}
\hline Parameters & Category & $\begin{array}{c}\text { Number } \\
\text { of cases (\%) }\end{array}$ \\
\hline \multirow{3}{*}{$\begin{array}{l}\text { Age } \\
\text { [years] }\end{array}$} & 20 to 88 & \\
\hline & Mean: 63 & \\
\hline & Median: 65 & \\
\hline \multirow[t]{2}{*}{ Gender } & Female & $95(40)$ \\
\hline & Male & $144(60)$ \\
\hline \multirow{3}{*}{$\begin{array}{l}\text { Tumor size } \\
{[\mathrm{cm}]}\end{array}$} & 0.45 to 19 & \\
\hline & Mean: 5.76 & \\
\hline & Median: 5 & \\
\hline \multirow{2}{*}{$\begin{array}{l}\text { Lymph node } \\
\text { metastasis }\end{array}$} & Yes & $161(67.4)$ \\
\hline & No & $78(32.6)$ \\
\hline \multirow[t]{2}{*}{ Lymphatic invasion } & Yes & $126(52.7)$ \\
\hline & No & $113(47.3)$ \\
\hline \multirow[t]{2}{*}{ Vascular invasion } & Yes & $36(15.1)$ \\
\hline & No & $203(84.9)$ \\
\hline \multirow[t]{2}{*}{ Perineural invasion } & Yes & $108(45.2)$ \\
\hline & No & $131(54.8)$ \\
\hline \multirow{2}{*}{$\begin{array}{l}\text { Status of surgical } \\
\text { margin }\end{array}$} & RO & $126(94.6)$ \\
\hline & $R 1-R 2$ & $13(5.4)$ \\
\hline \multirow[t]{4}{*}{ Tumor stage } & $\mathrm{T} 1$ & $53(22.2)$ \\
\hline & $\mathrm{T} 2$ & $51(21.4)$ \\
\hline & T3 & $100(41.8)$ \\
\hline & T4 & $35(14.6)$ \\
\hline
\end{tabular}

tric tissue (Figure 1). This result was statistically significant $(p<0.001)$.

To determine the status of mTOR activation, we evaluated by immunohistochemistry the phosphorylation status of mTOR using an antibody directed at the phosphorylated mTOR Ser2448 (p-mTOR), which recognizes the active form of mTOR [6].

p-mTOR positivity was observed in the membrane and cytoplasm of the GC cells, but not in the normal gastric mucosa or stromal cells (Figure 2).

In contrast, PTEN expression was weak and focal in the tumor cells, while in the normal gastric tissue this staining was strong and diffuse. PTEN staining was observed in the nuclei and cytoplasm of the cells (Figure 3).

Association between p-AKT, p-mTOR, and PTEN expression and clinicopathological features

High expression of $\mathrm{p}-\mathrm{AKT}$ protein was significantly associated with tumor size $(p<0.001)$, T3/
T4 tumors $(p<0.001)$, and presence of distant metastasis $(p=0.02)$. According to Lauren's GC classification, high expression of PTEN was associated with diffuse GC ( $p=0.007)$. The intestinal type was significantly associated with high expression of $p-m T O R(p=0.005)$. Indeed, significant correlations between high $p$-mTOR expression and GC with perineural invasion $(p=0.02)$ and T3/T4 stage were determined $(p=0.03)$.

\section{Influence of expression of $p-A K T, p-m T O R$, and PTEN on clinical outcome}

Kaplan-Meier survival curves were constructed using overall survival. The survival rate of the patients with a high p-AKT expressing GC was not different from those with low p-AKT expression. However, patients with high p-mTOR expression had a worse prognosis than those with low p-mTOR expressing GC ( $p=0.002)$. In contrast, patients with high PTEN expressing GC had a significantly better overall survival rate than patients with low PTEN expressing GC ( $p=0.008$, Figure 4).

The univariate analysis identified that all clinicopathological features, except patient age, significantly influenced patients' overall survival. The multivariate analysis by the Cox proportional hazards model identified that low expression of PTEN, high expression of $\mathrm{p}$-mTOR, advanced tumor stage, size, and diffuse type of GC were independent and significant determinants of worse prognosis for GC patients (Table II).

\section{Discussion}

Although a decline in the incidence of GC has been noted, it remains the sixth most common cancer worldwide [6]. The most efficient treatment for solid tumors is based on surgical intervention. The discovery of new chemotherapeutic agents that may improve the disease-free interval and overall survival of patients with GC remains a major challenge for researchers [7]. Although studies regarding the molecular alterations of GC have considerably increased in recent years, the results are still confusing and controversial. Previous studies have sought to identify the molecular alterations associated with the carcinogenesis steps of the major histological types of GC [8]. The use of molecular pathology techniques, such as immunohistochemistry, has helped to advance in this field [9].

The PI3K/AKT pathway is one of the most explored molecular pathways for identification of the therapeutic target, because it plays a central role in cellular proliferation and survival [5]. In the present study, we used immunohistochemistry to study PI3K/AKT pathway protein expression in a series of GCs and normal gastric tissues. 

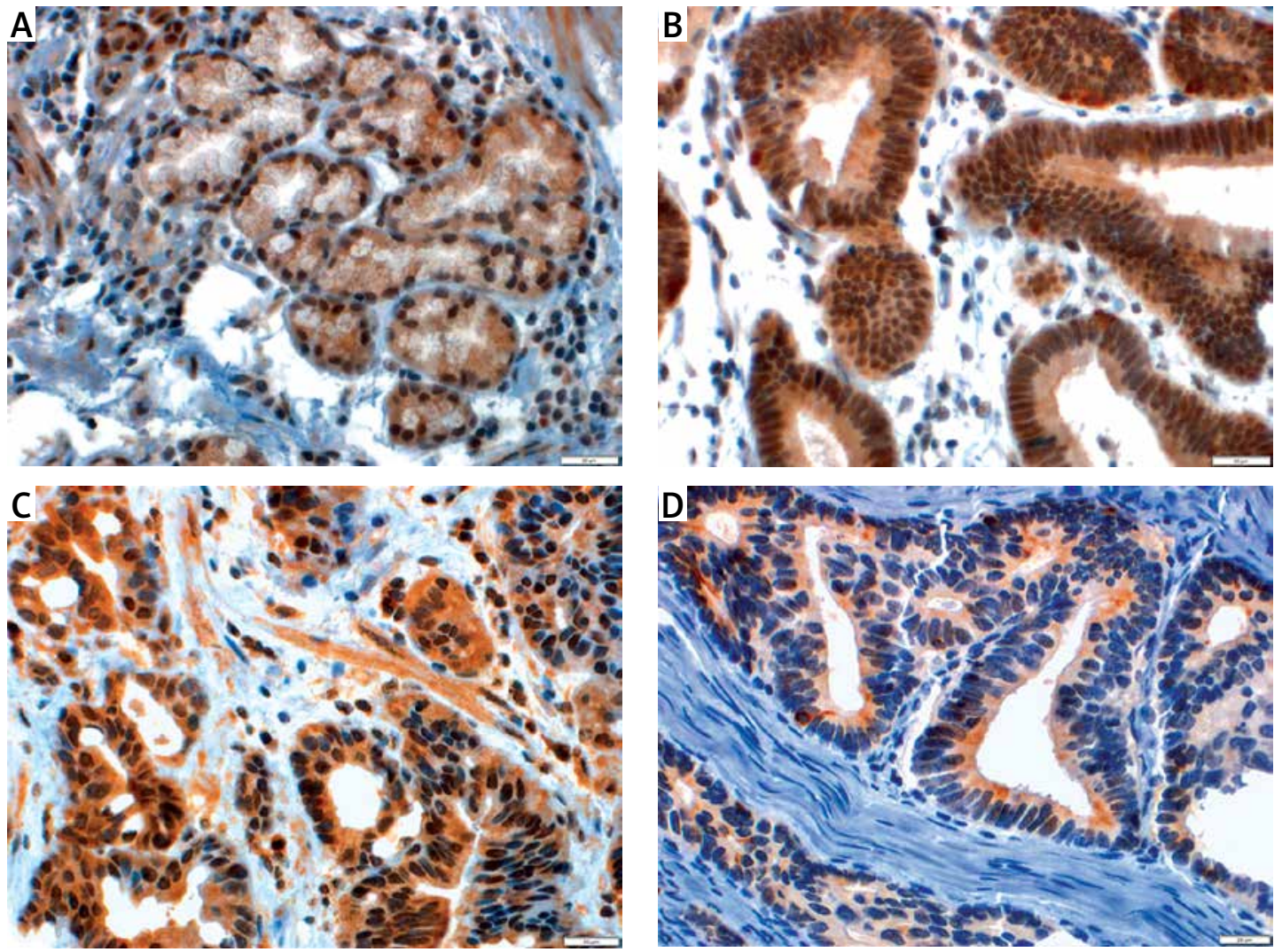

Figure 1. Immunostaining of p-AKT expression in the normal gastric tissues and tumor samples. A - Normal gastric tissue with low expression of p-AKT in the cells (original size 200x), B - Normal gastric tissue with high expression of p-AKT (original size 400x), C - Advanced gastric carcinomas (T3/T4) with high expression of p-AKT (original size 400x), D - Low expression of p-AKT in T2 gastric carcinoma (original size 400x). Positivity was observed in the nuclei and cytoplasm of the cells
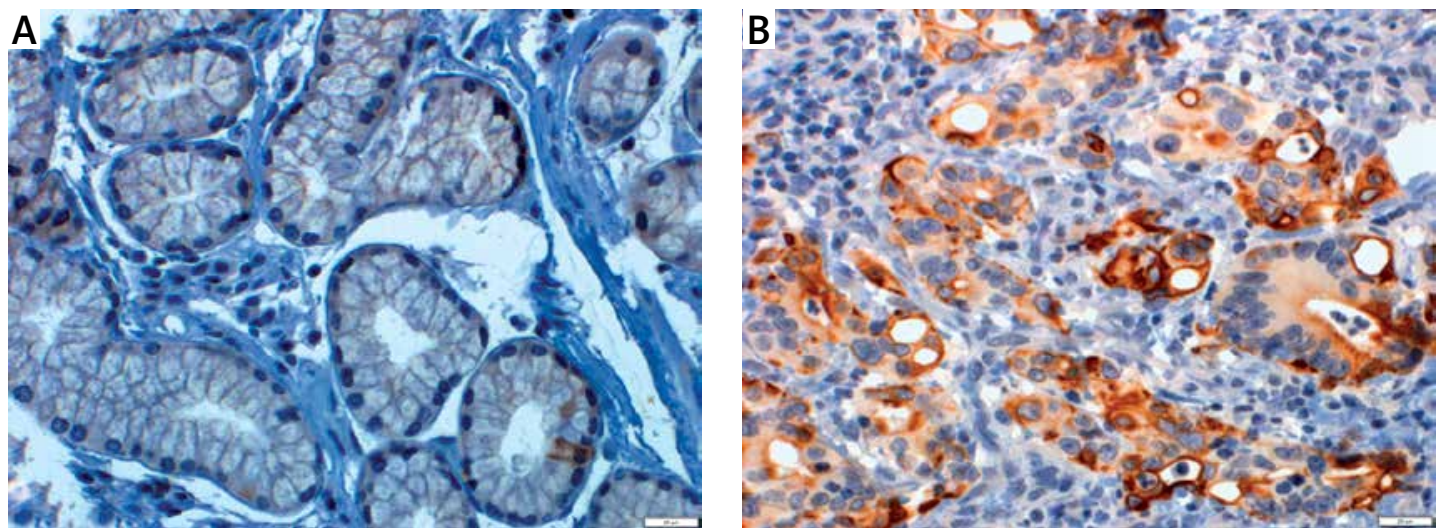

Figure 2. A - Normal gastric tissue negative for p-mTOR expression (original size 400x), B - High expression of p-mTOR in the cells of intestinal type of GC (original size 400x). The staining is primarily in the membrane and/or cytoplasm of tumor cells

Activated AKT has been linked to a poor prognosis in many cancers, and it has been shown that it can promote resistance to chemo- and radiotherapy. Recently, it has been demonstrated that high expression of p-AKT is associated with increased resistance to multiple chemotherapeutic agents in advanced GC patients [10].

Breast tumors with high p-AKT expression were associated with poor outcomes. Also, in colorectal cancer, high p-AKT expressing tumors were correlated with lymph node metastasis and advanced disease stage [11]. Conversely, increased p-AKT expression correlated with favorable outcomes in non-small-cell lung cancer [12]. In the present study, high p-AKT expressing GCs were associated with unfavorable clinicopathological findings, such as the largest size of tumors, advanced tumor stages, and presence of metastasis. Nam 
P.B.O. Chiappini, I.U.D. de Medeiros, L.G.C. Lima, J.H. Fregnani, S. Nonogaki, W.L. da Costa Jr, F.J.F. Coimbra, M.J. de Barros e Silva, C.A.L de Mello, C.A.L. Pinto, M.D. Begnami
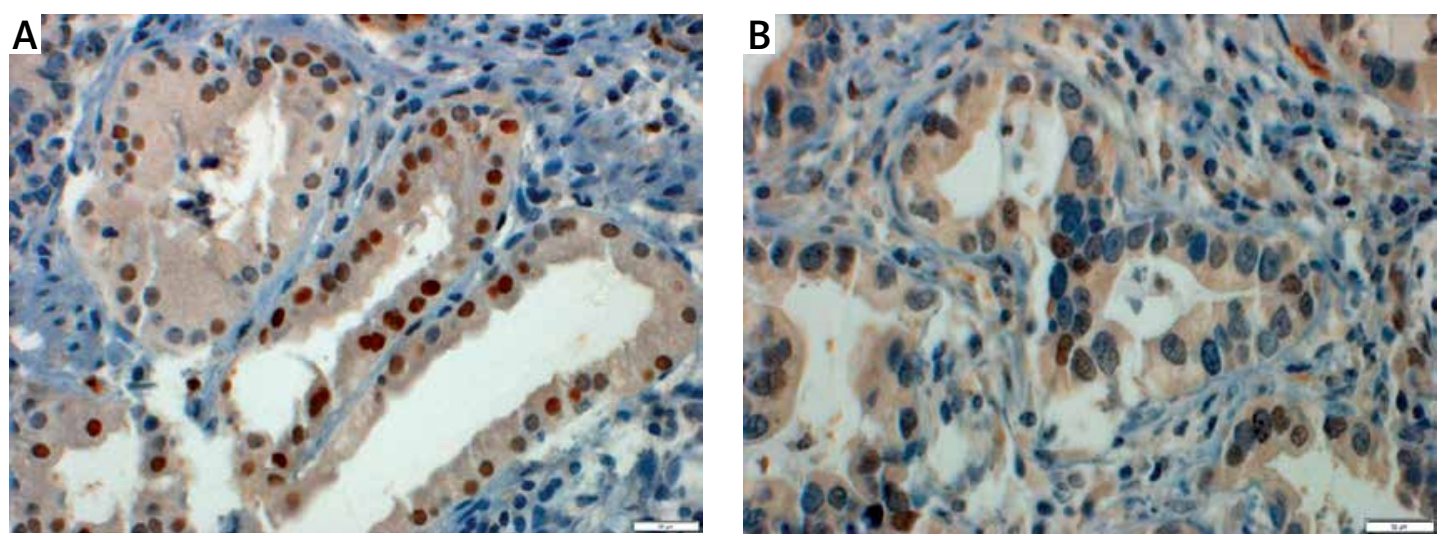

Figure 3. A - High PTEN expression in the cytoplasm and nuclei of the majority of normal gastric glands (original size 400x), B - Low expression of PTEN in GC (original size 400x)

A

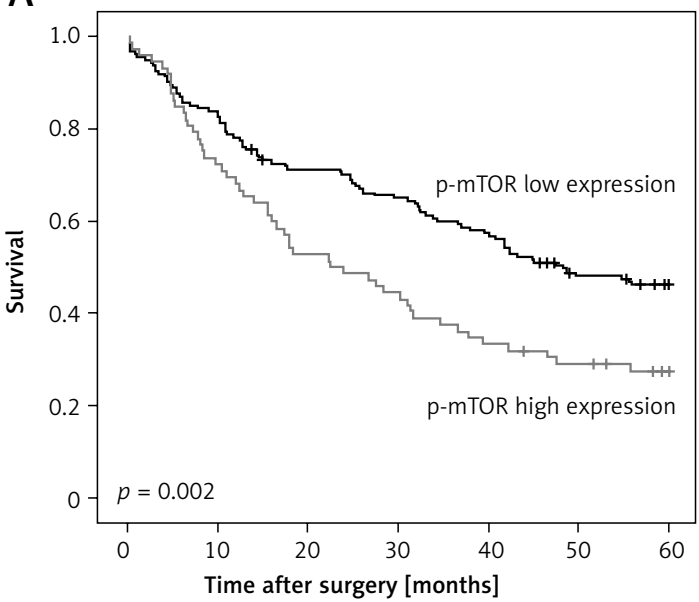

B

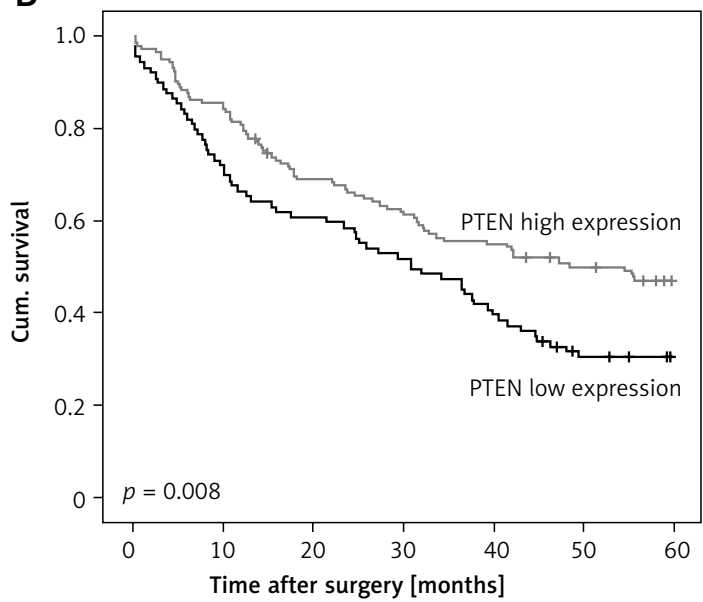

Figure 4. Kaplan-Meier curve for the overall survival of patients according to $p$-mTOR and PTEN expression. A Association of p-mTOR expression with survival time in 239 patients with gastric carcinomas. Median survival time was 20 months for patients with high expression of p-mTOR (compared with 50 months for patients with low p-mTOR expressing GC). The difference was significant in the log-rank test $(p=0.002)$. B - Median survival time was 30 months for patients with low expression of PTEN compared with 60 months for patients with high PTEN expressing GC. The difference was significant in the log-rank test $(p=0.008)$

Table II. Multivariate model for analysis of risk factors associated with death

\begin{tabular}{|lccccc|}
\hline Parameters & Category & $\boldsymbol{N}$ & HR & $\mathbf{9 5 \%} \mathrm{Cl}(\mathrm{HR})$ & $\boldsymbol{P}$-value \\
\hline PTEN staining & Low expression & 86 & 1.6 & $1.1-2.2$ & 0.012 \\
\cline { 2 - 6 } & High expression & 144 & 1.0 & Reference & \\
\hline p-mTOR staining & Low expression & 158 & 1.0 & Reference & 0.011 \\
\cline { 2 - 6 } & High expression & 72 & 1.6 & $1.1-2.3$ & \\
\hline Tumor stage & T1 & 48 & 1.0 & Reference & \\
\cline { 2 - 6 } & T2 & 49 & 2.1 & $1.02-4.3$ & 0.043 \\
\cline { 2 - 6 } & T3 & 98 & 5.4 & $2.8-10.4$ & $<0.001$ \\
\hline $\begin{array}{l}\text { Tumor gastric } \\
\text { involvement }\end{array}$ & Localized & 202 & 1.0 & Reference & 0.001 \\
\hline Tumor size & Diffuse & 28 & 2.3 & $1.4-3.7$ & \\
\hline
\end{tabular}

$N$ - number of cases, $H R$ - hazard ratio, $\mathrm{Cl}$ - confidence interval. 
et al. reported that gastric tumors with high $\mathrm{p}-\mathrm{AKT}$ expression were associated with few lymph node metastases but not followed by better overall survival in early staged tumors [13]. In contrast, Murayama et al. failed to demonstrate an association between p-AKT expression and clinicopathological features or survival [14]. These discrepancies may be explained by differences between the systems or antibodies used for p-AKT immunostaining and interpretation.

It has been reported that $\mathrm{p}$-mTOR is a powerful oncoprotein overexpressed in many types of cancer, including hepatocellular carcinoma [15], lung cancer [16], esophageal cancer [17], and breast cancer [18]. In GC, previous studies have demonstrated correlations between high p-mTOR expression and tumor invasiveness, presence of lymph node metastasis, advance stage, and worst rates for overall and disease-free survival $[14,19]$. In our study, we found high expression of p-mTOR mostly in T3/T4 GCs with perineural invasion. Furthermore, patients with high p-mTOR expressing GC had worse overall survival rates. These findings indicate that high expression of p-mTOR can be a marker of advanced and aggressive GCs. Several mTOR inhibitors drugs, including rapamycin/ everolimus, are being developed as anti-cancer agents against various types of malignancies [19], and they can be used in the treatment of GC patients with high expression of $p$-mTOR.

PTEN expression was observed in almost all normal gastric tissue studied. In contrast, this staining was weak and focal in the majority of the GC tumor cells. The loss of protein expression has frequently been associated with inactivation of the PTEN gene by mutation, deletion or promoter hypermethylation. It has been shown in various human cancer tissues [20-22]. The PI3K/AKT pathway is one of the most up-regulated pathways in neoplastic cells through mechanisms such as PTEN inactivation [23]. The loss of PTEN expression in the GC tumor cells observed in our study could be associated with downregulation of the PI3K/AKT pathway; however, our data do not support this correlation. Several studies that analyzed PTEN expression by immunohistochemistry showed nuclear localization of the protein expression in a wide variety of normal and tumoral tissues [24, 25]. The function of PTEN within the nuclei of the cells is the subject of ongoing investigations. It appears that the main PTEN activities are performed in the nuclei of the cells. However, the contribution of the regulatory mechanisms to the tumor suppressor activity of PTEN remains unclear [26].

In some settings, the effects of PTEN inactivation are predominantly manifested as changes in cell number, determined by the combined effects of proliferation and cell survival regulation. Deletion of PTEN associated with increased prolifera- tion, decreased apoptosis and tumorigenesis was noted in keratinocytes, prostatic epithelium, germ cells and hepatocytes [27].

The results of the present study, characterized by the loss of PTEN expression and higher expression of $p-A K T$ and $p-m T O R$ in the majority of the tumor cells, apparently are implicated in the carcinogenesis and progression of gastric carcinomas.

In conclusion, the identification of p-mTOR and PTEN expression as prognostic factors corroborates the identification and use of potential target drugs that could be more efficient for the treatment of these patients.

\section{Acknowledgments}

FAPESP: Grant 2010/16262-6.

\section{Conflict of interest}

The authors declare no conflict of interest.

\section{References}

1. Terry MB, Gaudet MM, Gammon MD. The epidemiology of gastric cancer. Semin Radiat Oncol 2002; 12: 111-27.

2. Weinstein IB, Joe AK. Mechanisms of disease: oncogene addiction: a rationale for molecular targeting in cancer therapy. Nature Clin Pract Oncol 2006; 3: 448-57.

3. Vivanco I, Sawyers CL. The phosphatidylinositol 3-kinase AKT pathway in human cancer. Nat Rev Cancer 2002; 2: 489-501.

4. Bellacosa A, Kumar CC, Di Cristofano A, Testa JR. Activation of AKT kinases in cancer: implications for therapeutic targeting. Adv Cancer Res 2005; 94: 29-86.

5. Tokunaga E, Oki E, Egashira A, et al. Deregulation of the Akt pathway in human cancer. Curr Cancer Drug Targets 2008; 8: 27-36.

6. Crew KD, Neugut Al. Epidemiology of gastric cancer. World J Gastroenterol 2006; 12: 354-62.

7. Anderson C, Nijagal A, Kim J. Molecular markers for gastric adenocarcinoma: an update. Mol Diagn Ther 2006; 10: 345-52.

8. Wang Z, Hao B, Yang Y, Wang R, Li Y, Wu Q. Prognostic role of SPARC expression in gastric cancer: a meta-analysis. Arch Med Sci 2014; 10: 863-9.

9. Begnami MD, Fregnani JH, Brentani $\mathrm{H}$, et al. Identification of protein expression signatures in gastric carcinomas using clustering analysis. J Gastroenterol Hepatol 2012; 27: 378-84.

10. Sasaki T, Kuniyasu H. Significance of AKT in gastric cancer (Review). Int J Oncol 2014; 45: 2187-92.

11. Itoh N, Semba S, Ito M, Takeda H, Kawata S, Yamakawa M. Phosphorylation of Akt/PKB is required for suppression of cancer cell apoptosis and tumor progression in human colorectal carcinoma. Cancer 2002; 94: 3127-34.

12. Shah A, Swain WA, Richardson D, et al. Phospho-akt expression is associated with a favorable outcome in nonsmall cell lung cancer. Clin Cancer Res 2005; 11: 2930-6.

13. Nam SY, Lee HS, Jung GA, et al. Akt/PKB activation in gastric carcinomas correlates with clinicopathologic variables and prognosis. APMIS 2003; 111: 1105-13.

14. Murayama T, Inokuchi M, Takagi $Y$, et al. Relation between outcomes and localisation of p-mTOR expression in gastric cancer. Br J Cancer 2009; 100: 782-8. 
P.B.O. Chiappini, I.U.D. de Medeiros, L.G.C. Lima, J.H. Fregnani, S. Nonogaki, W.L. da Costa Jr, F.J.F. Coimbra, M.J. de Barros e Silva, C.A.L de Mello, C.A.L. Pinto, M.D. Begnami

15. Sahin F, Kannangai R, Adegbola O, Wang J, Su G, Torbenson M. MTOR and P70 S6 kinase expression in primary liver neoplasms. Clin Cancer Res 2004; 10: 8421-5.

16. Schmid K, Bago-Horvath Z, Berger W, et al. Dual inhibition of EGFR and mTOR pathways in small cell lung cancer. Br J Cancer 2010; 103: 622-8.

17. Hirashima K, Baba Y, Watanabe M, et al. Phosphorylated mTOR expression is associated with poor prognosis for patients with esophageal squamous cell carcinoma. Ann Surg Oncol 2010; 17: 2486-93.

18. Bose S, Chandran S, Mirocha JM, Bose N. The Akt pathway in human breast cancer: a tissue-array-based analysis. Mod Pathol 2006; 19: 238-45.

19. LoPiccolo J, Blumenthal GM, Bernstein WB, Dennis PA Targeting the PI3K/Akt/mTOR pathway: effective combinations and clinical considerations. Drug Resist Updat 2008; 11: 32-50

20. Jang KS, Song YS, Jang SH, et al. Clinicopathological significance of nuclear PTEN expression in colorectal adenocarcinoma. Histopathology 2010; 56: 229-39.

21. Hou G, Lu Z, Liu M, Liu H, Xue L. Mutational analysis of the PTEN gene and its effects in esophageal squamous cell carcinoma. Dig Dis Sci 2011; 56: 1315-22.

22. Gonzalez-Angulo AM, Ferrer-Lozano J, Stemke-Hale K, et al. PI3K pathway mutations and PTEN levels in primary and metastatic breast cancer. Mol Cancer Ther 2011; 10: 1093-101.

23. Yang L, Kuang LG, Zheng HC, et al. PTEN encoding product: a marker for tumorigenesis and progression of gastric carcinoma. World J Gastroenterol 2003; 9: 35-9.

24. Zheng HC, Li YL, Sun JM, et al. Growth, invasion, metastasis, differentiation, angiogenesis and apoptosis of gastric cancer regulated by expression of PTEN encoding products. World J Gastroenterol 2003; 9: 1662-6.

25. Kim SJ, Lee HW, Baek JH, et al. Activation of nuclear PTEN by inhibition of Notch signaling induces G2/M cell cycle arrest in gastric cancer. Oncogene 2016; 35: 251-60.

26. Xu WT, Yang Z, Lu NH. Roles of PTEN (Phosphatase and Tensin Homolog) in gastric cancer development and progression. Asian Pac J Cancer Prev 2014; 15: 17-24.

27. Wen YG, Wang Q, Zhou CZ, Qiu GQ, Peng ZH, Tang HM. Mutation analysis of tumor suppressor gene PTEN in patients with gastric carcinomas and its impact on PI3K/AKT pathway. Oncol Rep 2010; 24: 89-95. 